
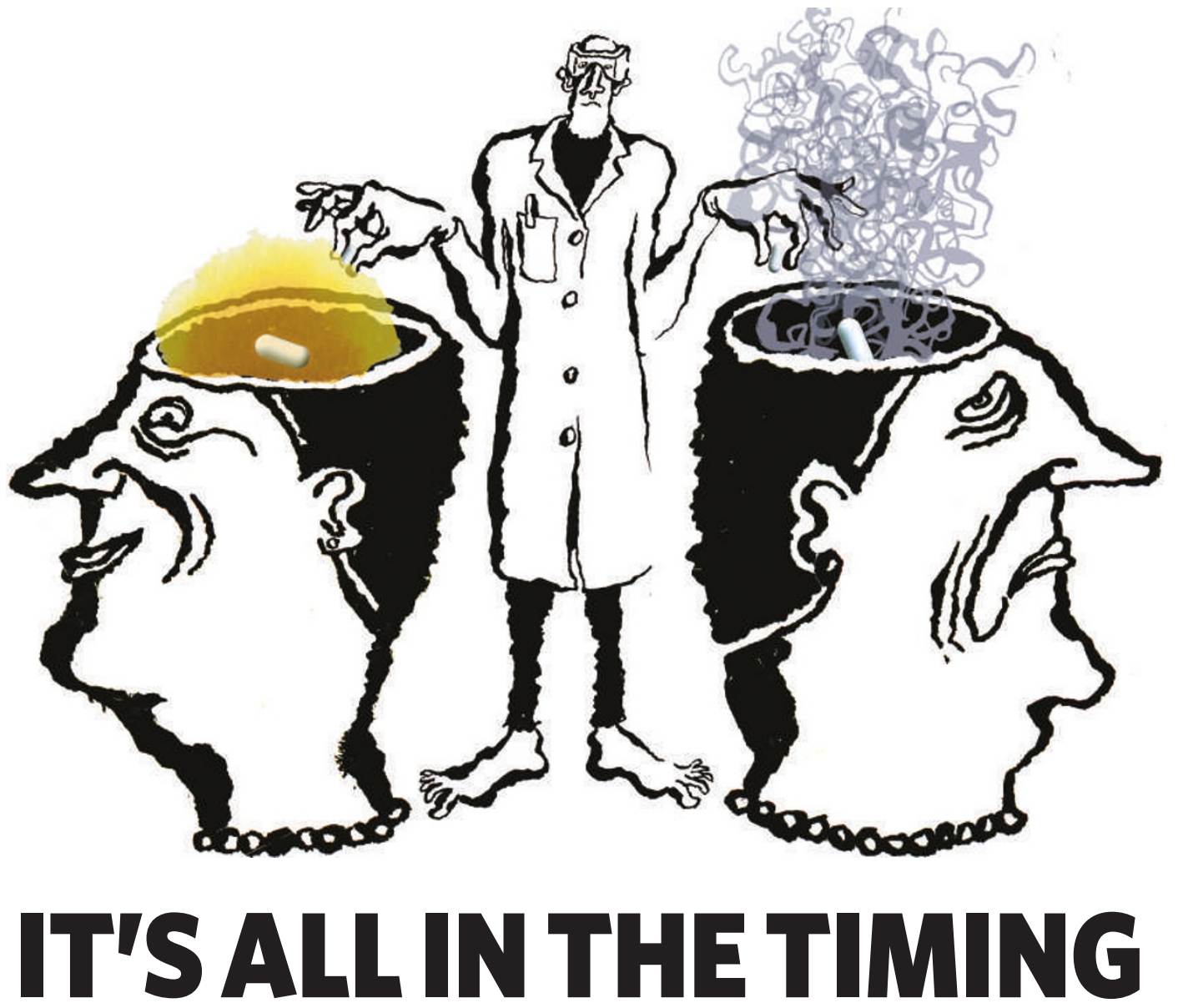

Taking hormones to replace those lost during menopause helps many women with their symptoms, yet it may also cause cognitive decline. Could the age at which hormones are taken determine whether they will be beneficial or harmful? Tom Siegfried reports.

$\Lambda$ geing female brains are perplexing, and not just to ageing husbands. Male and female neuroscientists are confused about the role that sex hormones play in mental decline after menopause.

Fuzzy memory and other mental maladies often accompany menopause, when natural production of steroidal sex hormones - mostly oestrogens - diminishes. Until a few years ago, evidence suggested that replacement of the lost hormones could alleviate memory problems and even protect against dementia later in life.

But in 2003, a large clinical trial performed in the United States reported that a popular type of hormone replacement therapy (HRT) did not improve cognition - and actually raised the risk of dementia ${ }^{1,2}$. That result baffled many scientists, as it contradicted both animal experiments and preliminary human studies showing that HRT benefited the brain.

But a better understanding of the biology that governs the brain's response to HRT might help to explain these discrepancies. The latest research is revealing a neurochemical soap opera about the brain's relationship with the sex organs, and how that relationship turns turbulent as the body ages ${ }^{3}$.

The latest twist in the plot suggests that HRT can indeed aid the brain, especially if the tim- ing is right. In fact, when given to a relatively young and healthy brain, HRT could well protect it against later damage. But when given to a brain that is already in decline, it can make things worse. "Timing is everything," says Andrea Gore, a neuroendocrinologist at the University of Texas at Austin.

After all, the landmark study that indicated possible mental declines under HRT - part of the massive Women's Health Initiative - tested women aged 65 to 79 . That is long past the average age of menopause in the Western world, at about 51 years old. Women who start HRT around the time of menopause, however, might instead reduce their risk of dementia, says Peter Schmidt of the National Institute of Mental Health in Bethesda,

Maryland, who studies the brain's response to sex hormones.

"There may be a critical window within which, if oestrogen is started, it will have a beneficial effect on the brain," he said in October in Atlanta at a meeting of the Society for Neuroscience. "The time and age at which a person takes hormone therapy may predict the clinical outcome."

Nobody knows for sure whether such a

window exists, or precisely when it would be open. And even if the benefits to the brain are confirmed, the Women's Health Initiative study identified other hazards from HRT, such as an elevated risk of breast cancer. The findings drove a dramatic drop in the number of postmenopausal women using HRT. In 2001, by one estimate, about 15 million women in the United States received HRT; that number fell to about

"The time and
the age at which
a person takes
hormone therapy
may predict the
clinical outcome."
- Peter Schmidt

10 million after the trial reported on its dangers ${ }^{4}$.

Women are now generally advised to take HRT for as short a time as possible (if at all) and only for treatment of menopausal symptoms. So, for HRT to help the brain, new hormone replacement molecules will probably be needed that confer the benefits without the risks.

To help in the hunt for new therapeutic molecules, researchers have realized that a deeper understanding is needed of the brain's role in menopause. For decades, research into menopause focused on the ovaries, which start to lose their hormone-producing follicles at age 40-50. "It really puzzled me that there was very little interest in whether the brain may also play a primary role in the control of reproductive ageing," says Gore. 
But research in Gore's and several other laboratories has shown that menopause does not simply result from the ovaries' decline. It also encompasses complex cross-talk between these organs and the brain. Menopause occurs in stages, and hormone secretion fluctuates considerably during the 'menopause transition' - the time from the first changes in the activity of the ovaries to the year after the final menstrual period.

That hormone variability reflects the shifting action in a three-character play starring the hypothalamus, a small structure at the base of the brain (see graphic). Supporting actors include the pituitary gland and the gonads (in women, the ovaries); collectively, the three make up the hypothalamic-pituitary-gonadal (HPG) axis.

The activity along the HPG axis is thought to be driven by the hypothalamus. Neurons there produce a peptide hormone known as gonadotropin-releasing hormone, or GnRH, which travels to the nearby pituitary gland and stimulates it to secrete other hormones. In turn, these hormones circulate to the ovaries, which respond to this chemical chain-mail by releasing their own hormones that travel back to the brain.

In younger adults, the characters in the HPG axis play their parts robustly, communicating clearly to maintain the body's reproductive ability. Reproductive ability declines once the hypothalamus starts to falter in how it responds to feedback from the ovaries - especially to signals sent by the main type of oestrogen, known as oestradiol.
Several actors conspire to twist the plot at this point. Oestradiol messages cannot signal the GnRH neurons directly, but instead stimulate a type of receptor on neighbouring neurons in order to relay the signal. But that nearby receptor starts to exit the stage as the body ages. "The expression of that receptor changes between young and middle-aged animals," Gore notes.

\section{Early retirement}

The GnRH neurons themselves remain onstage, but no longer respond as effectively to the signals that do get relayed from their neighbours. The problem, Gore says, seems to be related to subtle alterations in receptors for glutamate - the courier for the signals - on the ageing $\mathrm{GnRH}$ neurons.

Other players in the drama also diverge from the original script. Chemical cues sent to the hypothalamus from the ovaries change with age. In fact, all three levels of the HPG axis are changed in ageing rats and monkeys. "The responsiveness of this hypothalamic network to ovarian input decreases with ageing," says Gore. "Here is where timing is everything."

For some reason, she says, the hypothalamus gets old while the rest of the brain remains middle-aged. That hypothalamic senescence can trigger menopause-related mental decline years before deficits in learning and memory would normally start to become evident.

When the hypothalamus loses interest in the messages from the ovaries, hormone production diminishes. But the loss of hormones can't be the only thing that causes mental deficits after

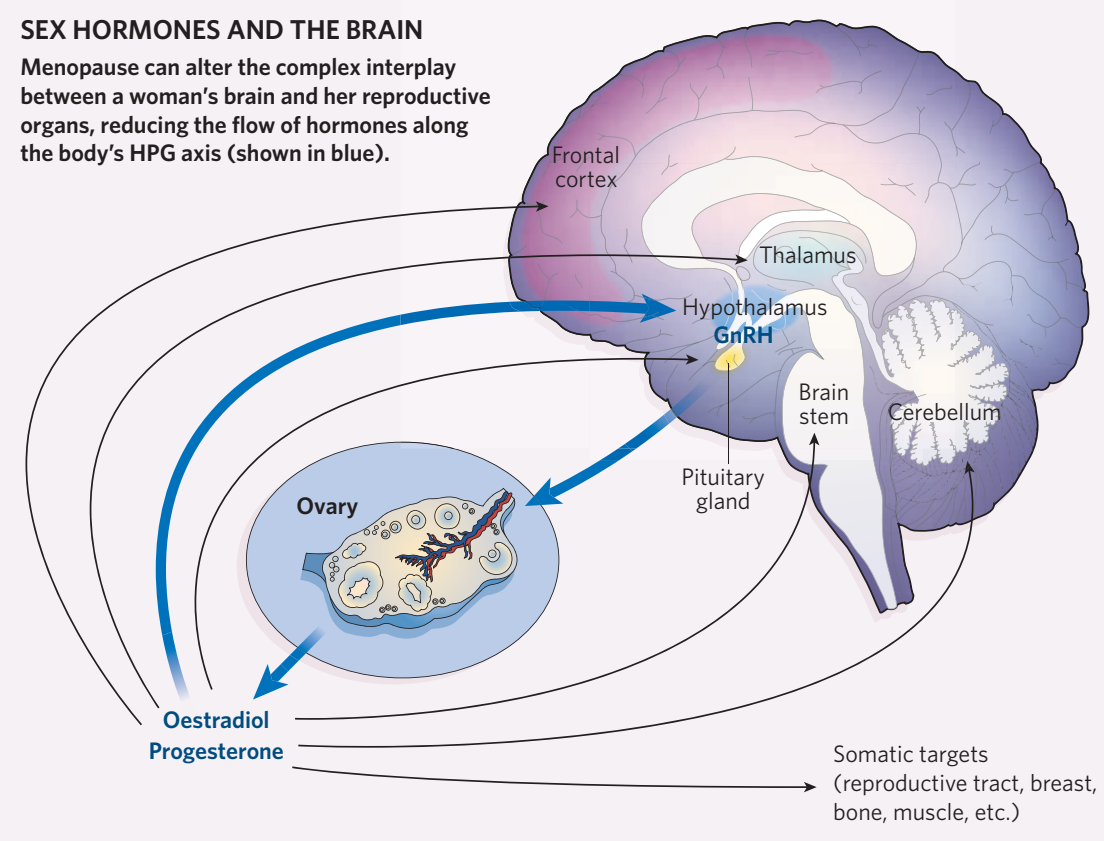

menopause. Young rhesus monkeys whose ovaries have been removed, depriving them of oestrogen, can still perform well on mental tests, says neuroscientist John Morrison of the Mount Sinai School of Medicine in New York City. "So they could counter the absence of oestradiol, whereas the aged animals could not." Older monkeys can, however, regain robust mental function when given oestradiol supplements.

Oestradiol apparently enhances memory skills by boosting the numbers of dendritic spines, which are sites on neurons where synapses, or connections, form. Morrison's research shows that oestradiol increases the density of synapses in the prefrontal cortex - the region of the brain responsible for the highest level of mental function.

To produce those benefits for ageing monkeys, Morrison and collaborators found, timing is doubly important. Besides administering oestradiol to monkeys near the time of menopause, researchers found the best results when injecting it at 21-day intervals, which mimicked the monkeys' natural hormonal cycle.

\section{On a whim}

Timing is clearly at the heart of the debate over HRT in women as well. The large clinical trial - called WHIMS, for the memorystudy part of the Women's Health Initiative - documented problems that stemmed from the commonly prescribed combination of oestrogen and progestogen. (Oestrogen alone is typically prescribed only for women who have had their uterus removed, as oestrogen alone raises the risk of uterine cancer.) An analysis of more than 4,000 women found that the rate of dementia was approximately doubled in elderly women on combination HRT compared with those on a placebo.

That surprising result led researchers to ponder whether HRT could be beneficial if administered to younger women, around the time of menopause. And studies examining oestrogen's actions within cells seem to support that suggestion.

Young, healthy cells are protected by oestrogen, says molecular neuropharmacologist Roberta Brinton of the University of Southern California in Los Angeles. But in cells already affected by disease (say, in the early stages of Alzheimer's), adding oestrogen accelerates the damage, her studies show ${ }^{5,6}$. That could explain why the earlier studies of younger women suggested that HRT helped the brain.

Within a neuron, Brinton says, oestrogen regulates the viability of mitochondria, the cell's energy-production centres. "If you damage mitochondria and you damage enough of them and damage them severely enough, the mitochondria release messengers that basi- 


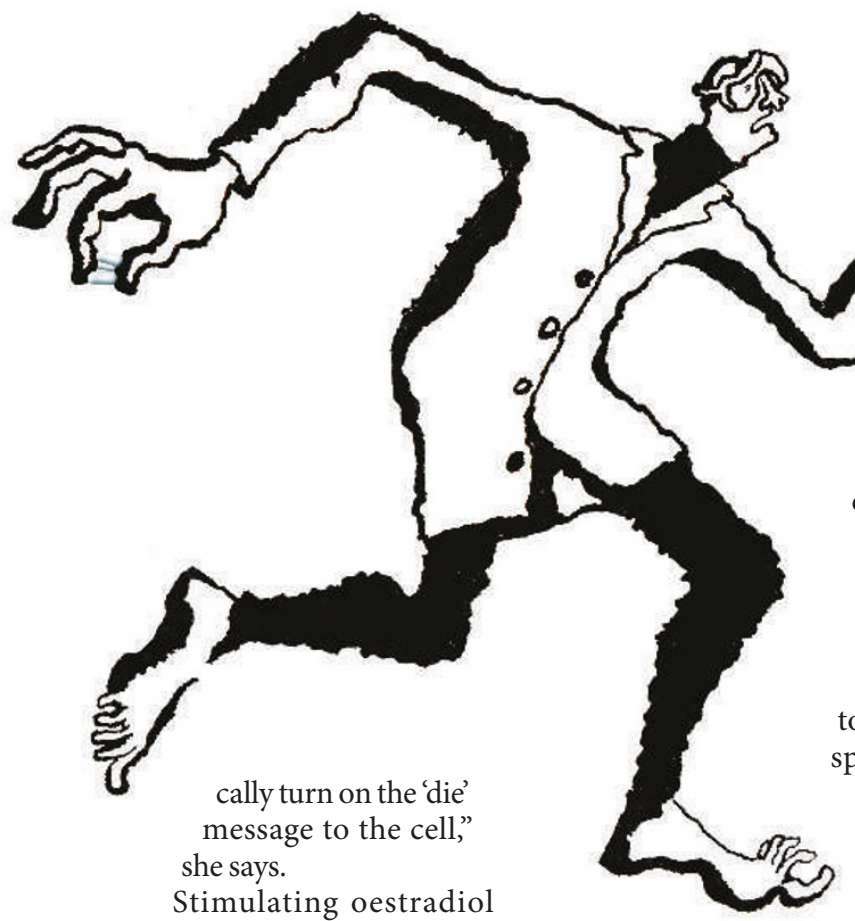

receptors on mitochondrial mem-

branes triggers a cascade of reactions that causes calcium to flood into a neuron. For a healthy neuron, that's not a problem, and in fact, the calcium influx initiates other chemical cascades that prevent mitochondria from signalling the cell to die. Older neurons, though, are not able to regulate calcium levels effectively, and therefore suffer even further if given oestrogen.

\section{Defensive driving}

"If you take a healthy cell and expose it to oestrogen, you create what I call a proactive defence survival state," Brinton says. Experiments show that such 'protected' cells can resist attacks from toxic substances such as free radicals or amyloid. "These cells have about a $25-50 \%$ survival advantage," she says.

On the other hand, for a damaged cell, oestrogen turns from a friend into an enemy. When experimenters expose a cell to toxic insults first, addition of oestrogen later leads to further degeneration ${ }^{5}$.

Brinton believes that women in the WHIMS study who developed Alzheimer's disease while taking oestrogen-progestogen supplements were probably in the early stages of Alzheimer's already. Replacement hormones then accelerated the disease.

“That's our hypothesis," she says. "You have to treat these cells in the brain at a time when they are healthy, when they have healthy calcium function, and that allows them to activate these mechanisms that lead to survival." So far, her results are based on studies of cultured neurons, but similar experiments have now started in a transgenic mouse model of Alzheimer's disease.

Further studies in Brinton's lab focus on the precise formulation of HRT, which typically and tested ${ }^{6}$.
— known as neuroSERMs - that would offer oestrogen's positive neurological benefits without the negative effects of standard HRT. Effective neuroSERMs would penetrate the blood-brain barrier and activate particular oestrogen receptors in the brain, without stimulating receptors responsible for deleterious effects in other tissues, such as in the breast or ovaries. Numerous neuroSERM candidate molecules are being developed

Still, for all the advances in mapping out the interplay of brains and hormones, many issues remain unresolved. For

"You have to treat
these cells in the
brain at a time when
they are healthy."
- Roberta Brinton

will not. The trick is to identify who belongs to which group. So a major priority of future research will be finding biomolecules that signal whether an individual is likely to benefit (or suffer) from hormone supplements. "Biomarkers are a critically important issue in all of ageing research," says Morrison. "We sorely need biomarkers to give us a reflection of brain changes for any treatment of age-related disorders."

\section{A complicated affair}

Investigations aimed at identifying such biomarkers will have to sort out many poorly understood biochemical complications. Oestradiol, for instance, operates on at least two receptors - alpha and beta - found at different sites in the brain. Add in the realization that the brain can produce its own oestrogen, as well as respond to oestrogen from the hormonal system, and ample avenues open up for new subplots in the hormone soap opera.

"You have to take into consideration the complexity of where the oestrogen receptor is," says Brinton. Oestrogen receptors are found not only on mitochondria, but also in a neuron's outer membrane and in its nucleus. And as Gore points out, the body has oestrogen receptors in other tissues too. "There are different tissues and different targets," she says, "and as we age our bodies may express a different assortment of these oestrogen receptors, even within a specific tissue."

And although oestradiol is the most important of the steroidal sex hormones in women, it doesn't work alone. Male sex instance, more work is needed to determine whether a window of opportunity exists during which people would benefit from HRT, and if so, when it would be best to start, and end, an HRT regimen. "I think nobody really knows the answer," says Schmidt. "It will be important to nail down what we mean by this critical window, and whether in fact it would suffice to take hormone therapy during that time."

Some researchers also think that a similar window of opportunity might exist to evade some of HRT's other possible dangers, particularly heart disease. Oestrogen's effect on atherosclerosis, for instance, will be examined by the new Kronos Early Estrogen Prevention Study, a five-year randomized trial to test oestrogen supplements in women who are within three years of their last menstrual period ${ }^{7}$. The results from the Women's Health Initiative study indicated a raised risk of heart disease for users of HRT, but for much older women.

Ultimately, researchers expect that some women will benefit from HRT whereas others hormones - the androgens - also circulate, and could affect how much oestradiol ends up acting within the brain, says Gore.

As for men, all the attention given to menopause may have misled them into thinking that they have nothing to worry about with respect to oestrogen and the ageing brain. But hormone and ageing issues may affect them as well. The male brain contains oestrogen receptors too, says Gore. "So there may be similar types of effects in men."

Tom Siegfried is a science writer in

Los Angeles.

1. Shumaker, S. A. et al. J. Am. Med. Assoc. 289, 2651-2662 (2003)

2. Rapp, S. R. et al. J. Am. Med. Assoc. 289, 2663-2672 (2003).

3. Morrison, J. H., Brinton, A. D., Schmidt, P. J. \& Gore, A.C. J. Neurosci. 26, 10332-10348 (2006).

4. Hersh, A. L., Stefanick, M. L. \& Stafford, R. S. J. Am. Med. Assoc. 291, 47-53 (2004).

5. Chen, S., Nilsen, J. \& Brinton, R. D. Endocrinology 147, 5303-5313 (2006).

6. Zhao, L., O'Neill, K. \& Brinton, R. D. Brain Res. Rev. 49, 472-493 (2005)

7. Manson, J. E. et al. Menopause 13, 139-147 (2006). 